\title{
Understanding Color Stability by Visualization of Pigment Dispersions in Paint Films
}

\author{
G.C. van Leerdam,* Maria Stjerndahl,** and Leif Karlson**
}

* AkzoNobel Research, Development and Innovation, P.O. Box 10, 7400 AA Deventer, TheNetherlands

** AkzoNobel Functional Chemicals, 44485 Stenungsund, Sweden

Besides protection and the addition of functionality (e.g. self-cleaning), the decoration of objects to improve their aesthetics is one of the most important functions for an applied paint film during its lifetime. Paints derive their color from addition of inorganic or organic [1] pigment particles to an organic binder. Generating the right color and effect (e.g. metallic look) for a paint film is a complex interplay of various factors and is, amongst others, affected by the pigment dispersion before, during and after its application as well as by the resistance of the complete film and the individual pigments to the weathering conditions (water, UV) encountered during the films lifetime. Besides the organic matrix, dispersing agents, thickeners and surfactants all influence the dispersion of the pigments in the fluid as well as the dried paint.

Considerable research effort in coatings industry is devoted to understanding the relation between color (effects) as well as the changes in color upon aging or drying and the micro-structure of the specific paint. In order to relate color effects to pigment dispersions, the combined use of microscopic and spectroscopic analytical techniques is very useful. This presentation will highlight specific examples in which the strengths of several of these advanced micro-analytical techniques have been combined in order to obtain a detailed picture on pigment dispersion and light-fastness in both model and real life paint films.

Generally, visualizing the distribution of inorganic pigments in a paint film does not suffer from many problems as the materials provide enough contrast in SEM or TEM [2], while phase identification can be done by EDX analysis, either used in local or in imaging mode.

Visualizing the distribution of organic pigments or additives in the organic binder is a much bigger challenge, due to their inherent lack of contrast in conventional electron microscopy. Frequently, a pigment specific characterization procedure has to be developed based on the pigments composition and/or the presence of specific functional groups. The ability to selectively map chemical functionalities makes spectroscopic techniques like EELS on the TEM (Fig. 1), ToF-SIMS imaging [3] and IR/Raman imaging value-adding tools in the visualization of pigment dispersions or elucidation of the origin of color fading. In occasions where analytical methods are not able to distinguish between organic phases, contrast can be created by chemical methods i.e. by selective staining with metal-containing stains (Fig. 2).

As most microscopic and spectroscopic studies are done on cross-sectioned paint films, sample preparation is a crucial factor for providing reliable information. Awareness of the risk of creating artifacts by sample preparation in microscopy and spectroscopy applications is needed to avoid wrong conclusions. 
References

[1] W. Herbst, K. Hunger, Industrial Organic Pigments: Production, Properties, Applications, Wiley-VCH, Weinheim, 2004

[2] S. Farrokhpay, G.E. Morris, D. Fornasiero, P. Self, J. Coat. Technol. Res. 3, (2006), 475.

[3] K. Keune, J. Boon, Anal.Chem. 76(5), (2004), 1374.

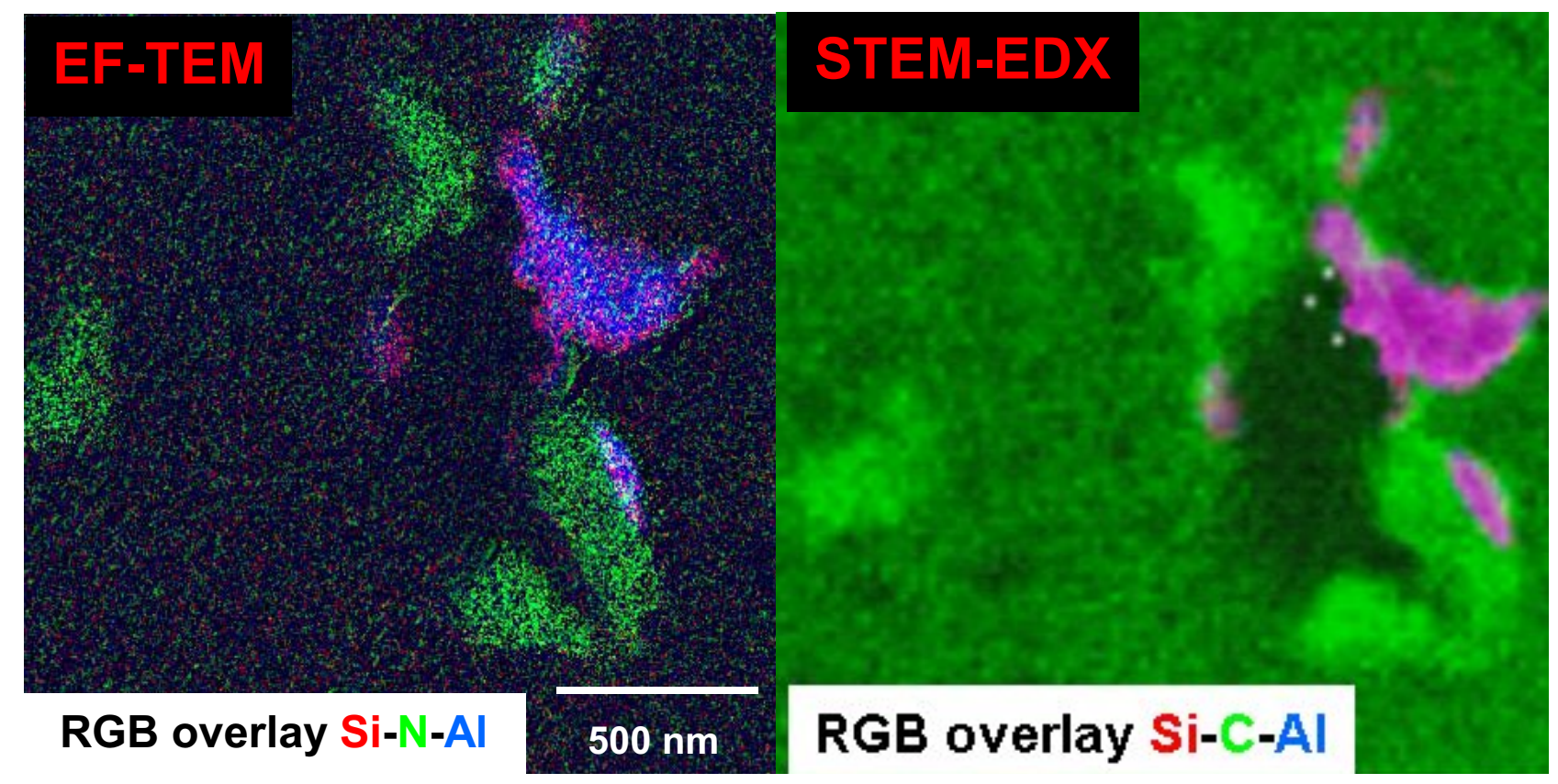

FIG. 1. Joint TEM/EELS and STEM/EDX chemical imaging of the same organic pigment particles and clay in paint (RGB overlays of characteristic elements).

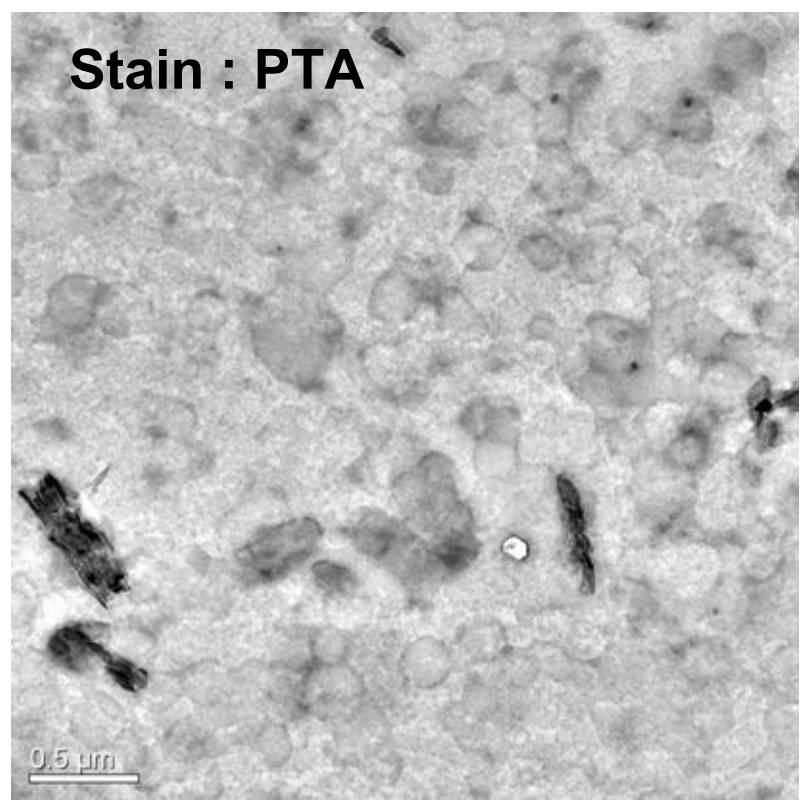

FIG. 2. TEM image of selectively stained latex in paint illustrating the film formation (phosphotungsten acid (PTA) stain). 